\title{
Sustained high prevalence of viral hepatitis and sexually transmissible infections among female sex workers in China: a systematic review and meta-analysis
}

Shu Su', Eric P. F. Chow ${ }^{2,3}$, Kathryn E. Muessig ${ }^{4,5}$, Lei Yuan ${ }^{6}$, Joseph D. Tucker ${ }^{4,7}$, Xiaohu Zhang ${ }^{8,9}$, Jiehui Ren ${ }^{10}$, Christopher K. Fairley ${ }^{2,3}$, Jun Jing ${ }^{9,2^{*}}$ and Lei Zhang ${ }^{1,2,3,9^{*}}$

\begin{abstract}
Background: The 1980's economic boom has been associated with a rapid expansion of China's sex industry over the past three decades. Consequently, the spread of sexually transmitted infections (STIS) and hepatitis infections among female sex workers (FSW) has become an important public health issue in China. This study identifies prevalence and risks of hepatitis and STIs in Chinese FSWs.

Method: Four electronic databases were searched for Chinese and English language peer-reviewed studies conducted between 01/2000-12/2011 that reported prevalence of hepatitis and STIs (excluding HIV) among Chinese FSW. Following the PRISMA guidelines, meta-analysis was used to estimate pooled prevalence and $95 \%$ confidence intervals for each infection.

Result: Three hundred and thirty nine articles (34 in English and 305 in Chinese) investigating 603,647 FSWs in 29 Chinese provinces were included in this review. Over the period 2000-2011, the seroprevalence of active hepatitis B and hepatitis C among FSW were $10.7 \%(7.3-15.5 \%)$ and $1.0 \%$ (0.7-1.3 \%), respectively. The most prevalent STI was human papillomavirus (HPV, 27.0 \% [10.1-55.1 \%]), followed by herpes simplex virus-2 (HSV-2, 15.8 \% [11.7-20.9\%]), chlamydia (13.7\% [12.1-15.4\%]), gonorrhoea (6.1\% [5.3-7.0\%]), syphilis (5.2\% [4.8-5.7\%]), genital warts (3.3\% [2.5-4.2\%]) and Trichomonas vaginitis (2.1\% [1.5-24.2\%]). Disease burden of both hepatitis and STI among FSW were concentrated in South Central and Southwest China. In particular, chlamydia and syphilis demonstrated a significant declining trend during the studied period $(P<0.05)$. Compared with the general Chinese population, FSW had significantly higher prevalence of all STIs except Trichomonas vaginitis. Further, compared to the general FSW population, HIV-positive FSW had significantly higher prevalence of syphilis, chlamydia, HSV-2 and Trichomonas vaginitis.
\end{abstract}

Conclusion: Prevalence of hepatitis and STIs remained high and mostly stable among Chinese FSW over the period of 2000-2011. Targeted STI and hepatitis surveillance and interventions should be strengthened among Chinese FSWs, especially those who are HIV-positive.

Keywords: Female sex worker, Sexually transmitted infection, Hepatitis, China, meta-analysis

\footnotetext{
* Correspondence: jingjun@tsinghua.edu.cn; lei.zhang1@monash.edu

${ }^{9}$ Research Center for Public Health, School of Medicine, Tsinghua University,

Beijing, China

${ }^{1}$ School of Public Health and Preventive Medicine, Faculty of Medicine,

Nursing and Health Sciences, Monash University, Melbourne, VIC, Australia

Full list of author information is available at the end of the article
} 


\section{Background}

Every year, sexually transmitted infections (STIs) affect more than 500 million people worldwide, posing a substantial threat to sexual and reproductive health of the world population [1]. While most STIs are curable with timely diagnosis and treatment, others lead to serious long-term effects including reproductive complications and death. Furthermore, individuals infected with STIs are more likely to contract or transmit HIV, and co-infection with hepatitis can complicate the treatment for HIV [2-4]. In response to these concerns, the World Health Organization has recommended prevention of STIs and hepatitis as a reproductive health priority $[5,6]$.

The 2013 statistics from the Chinese Ministry of Health reported a rapidly rising trend in STIs since the 1979 economic reform $[7,8]$. In the contexts of increasingly open attitudes toward commercial sex and higher disposable incomes, soliciting female sex workers (FSW) is increasing in China $[9,10]$. Commercial sex is illegal in China and subjected to harsh publishment, FSWs who are caught are sent to detention centres. Most Chinese FSWs sell sex in concealed venues and have established their own network to avoid being detected by the police. Given their opaque characters, frequent risk sexual activities and low awareness, Chinese FSWs are regarded as a highly at-risk group for STIs [11, 12]. Hepatitis $\mathrm{C}$ and $\mathrm{B}$ are also of concern among this group mainly due to higher rates of reported drug use (for HCV) and unsafe sexual behaviours (for HBV) among FSW as compared to the general population [13, 14]. Furthermore, transmission of STIs and hepatitis through male clients of FSW may have potential adverse impacts on the broader female population [15]. The estimated prevalence of HIV, syphilis, chlamydia and gonorrhoea were all higher among clients of female sex workers than among the general population, and a systematic review conducted in 2000-2012 demonstrated an significant increase in syphilis among male clients [16].

China's STI surveillance system routinely collects epidemic data of STIs only on syphilis and gonorrhoea. The data is collected by China CDC, but most of the data remain unpublished. Although numerous independent studies and an earlier systematic review [17] have reported STI and hepatitis infection burden among FSWs across China, these data have not been integrated to inform the overall geographic and temporal trends of STI infections among FSWs. In this study, we conducted a comprehensive data synthesis of hepatitis and STI epidemic information to inform the current disease burden, temporal trends and geographic distribution of STI infections among FSWs in China. This knowledge is important for informing HIV/STI health policies and designing evidence-based strategies for hepatitis and STI prevention among FSWs in China.

\section{Methods}

This meta-analysis was performed according to the PRISMA (Preferred Reporting Items for Systematic Reviews and Meta-Analyses) Statement issued in 2009 (Checklist S1).

\section{Literature and search strategy}

We searched one English database (PubMed) and three Chinese databases (Wanfang Data, VIP Chinese Journal Database and China National Knowledge Infra-structure) for studies conducted between January 1, 2000 to December 31, 2011 that reported the prevalence of STIs or hepatitis infections among FSW in mainland China.

The search included Medical Subject Headings (MeSH) terms for 'China, 'Chinese', 'CSW (commercial sex workers),' 'FSW', 'hepatitis', 'sexually transmitted diseases' and 'sexually transmitted infections', and other keywords associated with each STI: 'chlamydia,' 'Chlamydia trachomatis,' 'gonorrhoea,' 'Neisseria gonorrhoea,' 'syphilis', 'genital warts', 'hepatitis,' 'HBV', 'hepatitis B', 'HCV', 'hepatitis C', 'HSV', 'herpes simplex virus,' 'HPV', 'human papillomavirus' and 'trichomonas vaginitis'. Hand searching from the reference lists of the retrieved articles in the above databases was also included. Only articles published in Chinese or English language were included. Two reviewers (EPFC, XHZ) independently screened all retrieved abstracts from the four aforementioned databases to assess eligibility. Discrepancies were resolved by discussion with a third reviewer (LZ).

\section{Selection criteria \\ Type of studies}

Eligible study designs included quantitative epidemiological studies, including cohort and cross-sectional studies. The following types of publications were excluded: news reports, review articles, conference abstracts, mathematical modelling studies, clinical case studies, dissertations, studies with sample size less than 30 for general FSW or less than 10 for HIV-positive FSW, and STI prevalence reported without authenticated diagnosis (syphilis and hepatitis prevalence without serological test). Studies not conducted in mainland China were excluded.

\section{Type of participants}

We only included studies with FSW who self-reported having any commercial sexual activity (sell sex for money) in the past 12 months. There were no restrictions on age, marital status, educational level, ethnicity or residency. Studies that targeted HIV-positive FSW were included to investigate the prevalence of co-infection.

\section{Type of outcome measures}

We included studies that measured the prevalence of the most common STIs among FSW including syphilis, 
chlamydia, gonorrhoea, HPV/genital warts, HSV-2, and Trichomonas vaginitis. We also included HBV infection which is sexually transmitted and HCV infection which can be transmitted through injecting drug use. Studies were included if syphilis, chlamydia, gonorrhoea, HPV/ genital warts, HSV-2, Trichomonas vaginitis, $\mathrm{HBV}$ or $\mathrm{HCV}$ was diagnosed and confirmed by a serological testing approach. We included published studies in which HBV infection was defined by the presence of hepatitis $B$ surface antigen (HBsAg) as HBsAg is a serologic marker representing either acute or chronic HBV infection rather than due to other cases such as HBV immunity; HCV infection was diagnosed by the presence of anti-HCV antibodies; HPV infection was tested by PCR for any of the genital HPV types; chlamydia, gonorrhoea were tested by PCR, EIA or cell culture; syphilis was tested by specific treponemal serological method, rapid plasma regain or PCR and any positive result was considered positive; Trichomonas vaginitis was tested by wet mount and PCR; genital warts were clinically diagnosed based on the presence of warts; HSV-2 was diagnosed by the presence of type specific Immunoglobulin G (IgG) by EIA. Self-reported infections were excluded from this review.

\section{Quality assessment}

The authors assessed the methodological quality of every included study using an 8-item checklist tool for observational studies [18]. Based on the checklist guidelines, the quality of each item was categorized as either "High quality" ( 1 point) or "Low quality" ( 0 points) for a total possible score ranging between 0 and 8 . Studies with higher scores were regarded as higher quality.

\section{Data extraction}

Data were exported and entered into a Microsoft Excel database (Version 2010, Microsoft Corp., Redmond, WA, USA). Each study was given a unique ID number and the data collection form included information on: (1) Study design: location, sampling methods/venues, type of study, sample size, and study year; (2) epidemiology of STIs and hepatitis infections: prevalence estimates of STIs, HBV or $\mathrm{HCV}$, prevalence estimates of HPV gene type subgroups and prevalence estimates of sites and biomarkers of chlamydia and gonorrhoea.

\section{Statistical analysis}

Pooled estimates with $95 \%$ confidence intervals $(\mathrm{Cl})$ were calculated for the prevalence of each STI and hepatitis infection among Chinese FSW. These estimates were expressed as a percentage (number of infections divided by the number of individuals tested for the infection). Pooled odds ratios (OR) with $95 \% \mathrm{CIs}$ were calculated by comparing the pooled estimates between FSW and the general Chinese adult population. To obtain STI prevalence for various subgroups, we also conducted sensitivity analyses, and stratified by geographic region (North, Northeast, Northwest, East, South Central, Southwest), FSW venue type (entertainment venues, national sentinel sites, detaining education centres, VCT and others), sample size, language of the publication, study quality score, and study year (Additional file 1: Table S1 and S3). To estimate the risk of STI acquisition in HIV-positive FSW, the pooled $O R$ with a $95 \% C I$ was calculated by comparing the pooled STI prevalence estimates among HIV-positive FSW and general FSW. STI and hepatitis prevalence of the general population was estimated for the Chinese population of reproductive age (i.e. $>15$ years old) using data from national or large cross-sectional studies [19-24].

Heterogeneity across included studies was assessed using a chi-squared based Cochran's Q test and an $I^{2}$ test. If the data showed low heterogeneity $\left(p>0.05, I^{2}<50 \%\right)$, a fixed-effect model was used; otherwise, a random-effect model was used. Publication bias was tested by the Begg and Mazumdar rank correlation [25]. Geographical regions with first and second highest prevalence were defined as 'high prevalence' areas, the third and fourth ones were defined as 'moderate prevalence' whereas the fifth and sixth ones were defined as 'low prevalence' (Fig. 1b). The temporal trend of STIs was tested by chi-square trend test comparing the study periods 2000-2002, 2003-2005, 2006-2008, and 2009-2011 (Fig. 3). All meta-analyses were performed by using the Comprehensive MetaAnalysis (version 2.2, Biostat, Englewood, New Jersey).

\section{Results}

\section{Characteristics of the studies}

Out of the 3015 studies identified through initial searches, 339 studies (English: 34; Chinese: 305) were eligible and included in this review (Additional file 2). The majority of studies were cross-sectional observational studies ( $\mathrm{n}=$ 286). Eighteen studies reported on HBV, 97 on HCV, 215 on syphilis, 96 on chlamydia, 116 on gonorrhoea, 38 on HSV-2, 43 on Trichomonas vaginitis, 68 on genital warts and 4 on HPV, respectively (Table 1 ). From these published studies, 603,647 FSWs in 29 Chinese provinces were included in this review. Sample size ranged from 11 to 12,363 . The mean age of FSW was $24.2 \pm 2.4$, the average length of time working in commercial sex was 2.4 years. The estimated percentage of FSW who were married was $35.3 \%$, and $76.9 \%$ had only completed junior middle school education or below.

\section{Disease burden of hepatitis}

For studies conducted between 2000 and 2011, the estimated overall $\mathrm{HBV}$ and $\mathrm{HCV}$ prevalence among FSW were $10.7 \%$ (95 \% CI: 7.3-15.5 \%) and $1.0 \%(0.7-1.3 \%)$ (Table 1). Heterogeneities across the studies were substantial (HBV, $p<0.001 ; I^{2}=97.1 ; \mathrm{HCV}: p<0.001 ; I^{2}=$ 


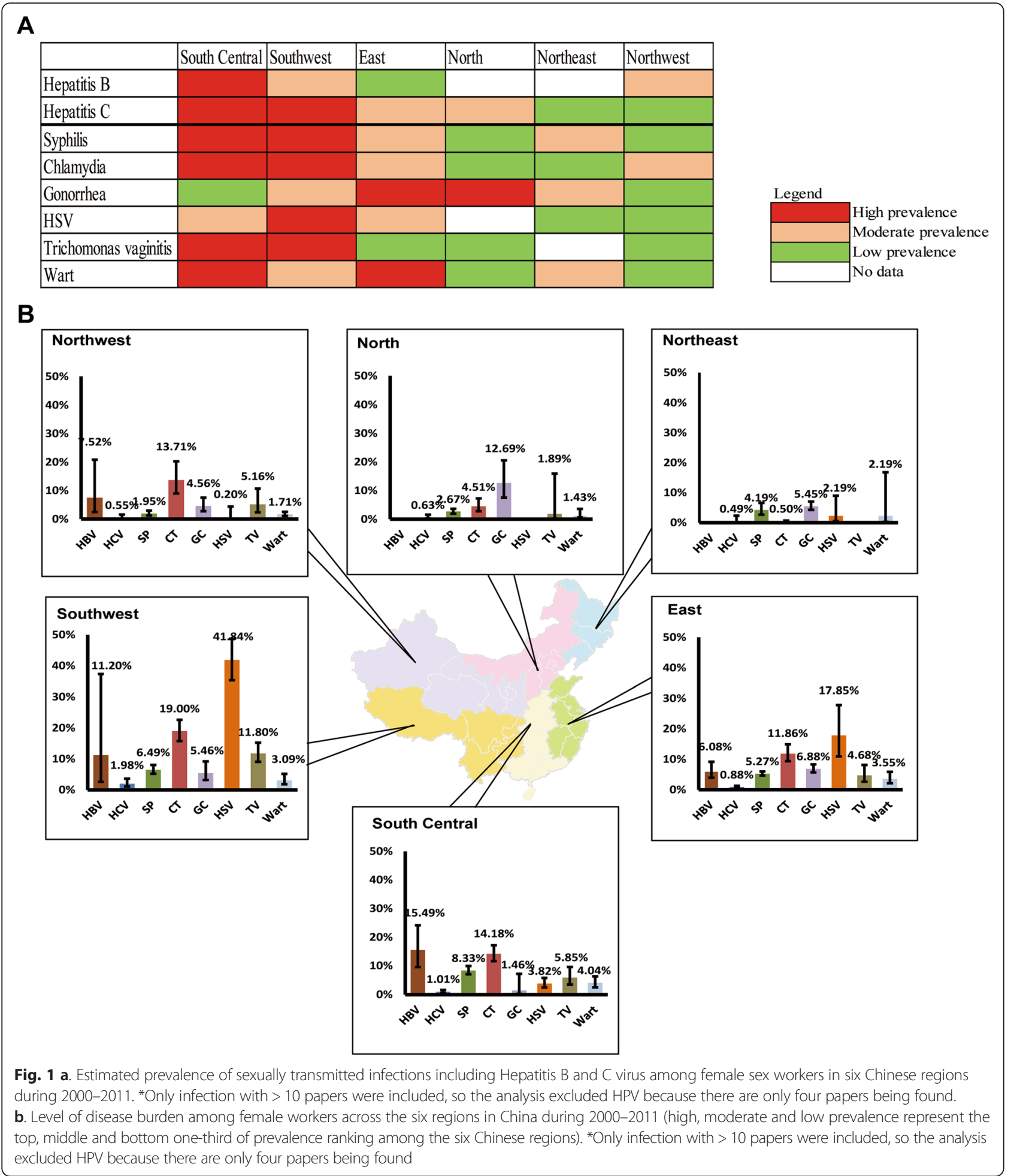

94.8). HBV disease burden varied substantially from $6.1 \%(4.0-9.2 \%)$ in the East to $15.5 \%(9.5-24.2 \%)$ in the South Central (Fig. 1a). No included studies reported HBV prevalence from FSW samples in the North and Northwest. In contrast, the Northwest had the lowest
HCV prevalence $(0.5 \%$ [0.2-1.6 \%]); while Southwest was the highest $(2.0 \%$ [1.1-3.6 \%], Fig. 1a). The temporal trends of both of them were not significant $(\mathrm{P}>$ 0.05). Compared to the general Chinese population, FSW had notably higher risk of $\mathrm{HBV}$ and $\mathrm{HCV}$ infection 
Table 1 Comparison of sexually transmitted infections prevalence between female sex workers and general female population in China

\begin{tabular}{|c|c|c|c|c|c|}
\hline Type of infection & $\begin{array}{l}\text { No. of studies } \\
\text { (No. of data points) }\end{array}$ & $\begin{array}{l}\text { Total No of infection/ } \\
\text { Sample size }\end{array}$ & $\begin{array}{l}\text { Pooled prevalence } \\
\text { among FSW }(95 \% \mathrm{Cl})\end{array}$ & $\begin{array}{l}\text { Background prevalence among } \\
\text { general population }(95 \% \mathrm{Cl})\end{array}$ & Odds ratio $(95 \% \mathrm{Cl})$ \\
\hline HBV & $18(24)$ & $848 / 7,614$ & $10.71(7.30-15.48)$ & $6.40(4.45-9.08)^{*}[26]$ & 1.75 (1.60-1.92) \\
\hline $\mathrm{HCV}$ & 97 (149) & $1,366 / 114,722$ & $0.96(0.73-1.25)$ & $0.12(0.05-0.27)^{*}[20]$ & 7.99 (3.58-17.83) \\
\hline Syphilis & 215 (377) & $13,507 / 245,455$ & $5.24(4.83-5.69)$ & $0.37(0.20-0.65)^{*}[21]$ & 14.89 (10.86-20.40) \\
\hline HSV-2 & $38(57)$ & $9,529 / 30,345$ & $15.77(11.74-20.85)$ & $5.80(3.82-7.78)^{*}[22]$ & $3.05(2.12-4.39)$ \\
\hline Trichomonas vaginitis & $43(65)$ & $2,271 / 24,381$ & $2.12(1.46-24.21)$ & $2.52(1.92-3.31)^{* *}[22]$ & $0.83(0.51-1.35)$ \\
\hline Chlamydia & $96(125)$ & $7,983 / 56,548$ & $13.66(12.11-15.37)$ & $2.60(1.60-4.10)^{* *}[23]$ & $5.95(4.42-8.01)$ \\
\hline Cervix & $41(78)$ & $5,503 / 33,231$ & $13.56(11.65-15.73)$ & - & - \\
\hline Vaginal & $3(4)$ & $402 / 872$ & $36.80(19.56-58.23)$ & - & - \\
\hline$P C R$ & $48(74)$ & $5,839 / 31,695$ & $15.48(13.70-17.45)$ & - & - \\
\hline Rapid test & $16(19)$ & $2,144 / 24,853$ & 10.28(8.20-12.83) & - & - \\
\hline Gonorrhea & $116(149)$ & $3,723 / 62,430$ & 6.07 (5.29-6.95) & $0.16(0.06-0.39)^{* *}[23]$ & $40.32(12.99-125.18)$ \\
\hline Cervix & $51(74)$ & $2,523 / 33,493$ & $6.88(5.68-8.31)$ & - & - \\
\hline Vaginal & $7(9)$ & $311 / 1,806$ & $12.69(7.52-20.60)$ & - & - \\
\hline PCR & $28(55)$ & $2,015 / 23,179$ & 8.33(6.83-10.13) & - & - \\
\hline Culture & $52(64)$ & $1,417 / 28,143$ & $5.62(4.51-6.97)$ & - & - \\
\hline Wart & $68(76)$ & $1,111 / 39,862$ & $3.27(2.53-4.21)$ & $0.06(0.01-0.07)^{* *}[20]$ & $52.96(26.42-106.15)$ \\
\hline HPV (any) & $4(4)$ & $531 / 1,631$ & $27.04(10.07-55.09)$ & $16.80(16.50-17.10)^{* *}[24]$ & $1.84(1.64-2.06)$ \\
\hline Gene type 16 & $3(3)$ & $142 / 1,143$ & $14.08(4.13-38.40)$ & $3.62(0.82-14.62)^{*}[61,62]$ & 4.39 (3.20-6.03) \\
\hline Gene type 52 & $3(3)$ & $150 / 1,143$ & $12.53(6.36-23.30)$ & $2.62(1.75-3.48)^{*}[61,62]$ & $5.32(3.72-7.63)$ \\
\hline Gene type 58 & $2(2)$ & $115 / 1,098$ & $12.12(2.65-41.11)$ & $1.59(1.07-2.36)^{*}[61,62]$ & $8.60(5.53-13.38)$ \\
\hline Gene type 33 & $3(3)$ & $75 / 1,143$ & $8.25(2.61-23.19)$ & $1.04(0.63-1.72)^{*}[61,62]$ & $8.48(4.96-14.48)$ \\
\hline Gene type 31 & $2(2)$ & $59 / 1,098$ & $5.05(3.84-42.25)$ & $2.09(1.44-3.02)^{*}[61,62]$ & $2.46(1.58-3.82)$ \\
\hline Multiple type & $2(2)$ & $216 / 1,098$ & $23.57(6.08-59.47)$ & - & - \\
\hline Single type & $2(2)$ & $328 / 1,098$ & $29.9(27.3-32.7)$ & - & - \\
\hline
\end{tabular}

* refers to $p<0.05$

** refers to $p<0.01$

$(\mathrm{HBV}:$ OR $=1.8[1.6-1.9] ; \mathrm{HCV}: \quad \mathrm{OR}=8.0 \quad[3.6-17.8])$ (Table 1).

\section{Disease burden of sexually transmitted infections}

Meta-analysis indicated that the most common STI among FSW was HPV, with a pooled prevalence of $27.0 \%$ (10.1$55.1 \%)$. Notably, genotype HPV16 (14.1 \% [4.1-38.4\%]) was the most frequently identified sub-type. About $23.6 \%$ (6.1-59.5 \%) of FSW were infected with multiple HPV subtypes. HSV-2 ranked second reaching $15.8 \%$ (11.7-20.9\%) during 2000-2011, followed by Chlamydia at 13.7 \% (12.1$15.4 \%)$. Subgroup analyses indicated that Chlamydia prevalence substantially varied by anatomical sites, ranging from $13.6 \%$ (11.7-15.7 \%) checked by cervical smear to $36.8 \%$ (19.6-58.2 \%) checked by vaginal swab. The pooled prevalence estimates of syphilis, genital warts and Trichomonas vaginitis were $5.2 \%(4.8-5.7 \%), 3.3 \%(2.5-4.2 \%)$ and $2.1 \%$ (1.5-24.2\%), respectively (Additional file 1: Table S2).

Risk of STIs among FSW was far higher than in the general population. The odds ratio (OR) for having genital warts among FSW was 81.4 (40.6-163.2) compared to the general population. This was followed by gonorrhoea (OR 40.3 [13.0-125.2]) and syphilis (OR 14.9 [10.9-20.4]) (Table 1). The prevalence of STIs was also higher among HIV-infected FSW compared to boarder FSW. Based on available data among HIV-positive FSW, prevalence of HSV-2, Chlamydia, Trichomonas vaginitis and syphilis were $83.2 \%$ (75.6-88.8 \%), $20.2 \%$ (14.3-27.7\%), $19.7 \%$ (12.3-30.2 \%) and $15.2 \%(8.3-26.4 \%)$, respectively. These corresponded to 27.2 (17.0-43.5), 2.2 (1.4-3.3), 11.5 (5.723.0) and 4.1 (2.8-6.0) higher risk comparing to the broader FSW population (Table 2).

\section{Temporal and geographical trend of STIs}

Among sampled FSW, several STIs demonstrated a temporal declining trend. Over the study period (2000 to 2011), reports of Chlamydia declined from $16.9 \%$ (6.6$35.1 \%)$ in $2000-2002$ to $10.7 \%(7.7-14.9 \%)$ in 2009$2011(\mathrm{p}=0.02)$ while reports of syphilis declined from $8.4 \%(4.9-14.4 \%)$ to $4.1 \%(3.2-5.5 \%)(\mathrm{p}<0.01)$. The 
Table 2 Comparison of sexually transmitted infections prevalence between HIV-positive female sex workers and general female sex workers in China

\begin{tabular}{llllll}
\hline Type of infection & $\begin{array}{l}\text { No. of studies } \\
\text { (No. of data points) }\end{array}$ & $\begin{array}{l}\text { Total No of infection/ } \\
\text { Sample size }\end{array}$ & $\begin{array}{l}\text { Pooled prevalence among } \\
\text { HIV-positive FSW (95 \% Cl) }\end{array}$ & $\begin{array}{l}\text { Pooled prevalence } \\
\text { among FSW (95 \% Cl) }\end{array}$ & Odds ratio (95 \% Cl) \\
\hline Chlamydia & $2(2)$ & $28 / 139$ & $20.21(14.33-27.72)$ & $13.66(12.11-15.37)$ & $2.15(1.42-3.26)$ \\
HSV-2 & $2(2)$ & $107 / 128$ & $83.22(75.60-88.82)$ & $15.77(11.74-20.85)$ & $13.6(8.4-22.2)$ \\
Syphilis & $5(5)$ & $27 / 210$ & $15.22(8.26-26.36)$ & $5.24(4.83-5.69)$ & $4.13(2.83-6.02)$ \\
Trichomonas vaginitis & $1(1)$ & $15 / 76$ & $19.74(12.26-30.20)$ & $2.12(1.46-24.21)$ & $11.48(5.73-22.99)$ \\
\hline
\end{tabular}

average annual decline in prevalence of chlamydia and syphilis were $9 \%(1-16 \%)$ and $2 \%(0-5 \%)$ respectively (Fig. 2). Temporal trends of other STIs did not show significant variations.

Southern Chinese regions had greater risk of STIs. In particular, FSW in the Southwest had high prevalence of HSV-2 (41.8 \% [35.3-38.7 \%]), Chlamydia (19.0 \% [15.8$22.6 \%]$ ) and Trichomonas Vaginitis (11.8 \% [9.1-15.2 \%]) (Fig. 1a). The South Central region shared a similar disease burden of STIs as seen in the Southwest but had the highest nationwide prevalence of syphilis and genital warts (8.3\% [7.0-10.0\%] and 4.0\% [2.6-6.3\%], respectively). Prevalence of gonorrhoea among FSW was highest in the North (12.7 \% [7.5-20.6 \%]) and East (6.9 \% [5.7-8.3 \%]) regions (Fig. 1a). Overall, the prevalence of most STIs were low to moderate among FSW in the northern Chinese regions (Fig. 1b).

\section{Heterogeneities and risk of bias}

Heterogeneities were significant in most of our metaanalyses (Additional file 1: Table S2). Larger sample size $(\geq 400)$ tended to result in higher prevalence estimates (in all STIs except HSV-2). Prevalence of syphilis (8.0\% [7.4-10.1 \%]), Trichomonas Vaginitis (13.2 \% [7.9$21.1 \%]$ ) and gonorrhoea (7.9 \% [5.9-10.6 \%]) were substantially higher among FSW sampled from detention centres than among those sampled from entertainment venues $(p<0.05)$. Study quality score was not associated with prevalence estimates for any infections except HSV-2 (Additional file 1: Table S2).

\section{Discussion and conclusions}

This meta-analysis has consistently demonstrated higher STI and viral hepatitis burden among FSWs in South Central and Southwest China compared to other Chinese regions. Compared with a previous similar systematic review [17], our study represents a significant extension in the investigation of temporal and geographical trend of STIs among FSW in reference to the general population. Apart from chlamydia and syphilis, prevalence levels of most STIs and hepatitis infections remain stable over the studied period. Compared with the general Chinese population, FSW had a significantly higher risk of STI and hepatitis infection. Similarly, HIV-positive FSW were more likely to have STIs than the broader FSW population.

Our study reported a national HBV prevalence of $10.7 \%$ (HBsAg active) among Chinese FSW but with distinct regional differences. This prevalence level is higher than that of the general Chinese population (7.9\% in 2010, [26]) and the prevalence $(7.18 \%)$ reported by China CDC in 2006 [27]. Among all Chinese regions, the finding that South Central China (15.5\%) has shown the highest prevalence is consistent with the prevalence in the general Chinese population $[13,19]$. HBV transmission in China is primarily through mother-to-child transmission [28, 29], but transmission by sex and injection sharing has also been reported in high-risk population groups [29]. The high HBV disease burden in Southern China is likely due to earlier onset of both the epidemic [30-32]. Also of note, China's national HBV vaccination programme for newborns was only introduced in 1992 [33]; many of our studied subjects (average age 24.2) may have been born before the start or scale-up of the vaccination program. Vertical transmission may be the dominant route of transmission of HBV in FSW, but high risk sexual behaviours of FSW has also substantially added to the risk of HBV infection in comparison with the general Chinese females. Targeted interventions, orchestrated by China CDC and local NGOs, should be implemented in FSW to improve awareness and reduce further HBV transmission via sexual means and vertical transmission.

Southwest China has reported the highest prevalence of HCV (2.0\%). Southwest China has faced illicit drug trafficking issues due to its close proximity bordering the 'golden triangle', a well-known area for heroin production, manufacture and distribution in Southeast Asia [13]. It hosts estimated 3 million PWID and provides $42.7 \%$ global seizures in heroin in 2013 [34]. Prevalent injection drug use may be the underlying reason for $\mathrm{HCV}$ transmission among FSW given that sexual transmission via heterosexual sex is rare [14, 35]. Among FSWs who use drugs, selling sex for drugs is notably more common in southern China than others regions [36, 37]. In order to alleviate the disease burden of $\mathrm{HCV}$, specialised behavioural interventions for FSW with overlapping injecting and risk sexual behaviours should be designed and implemented. 


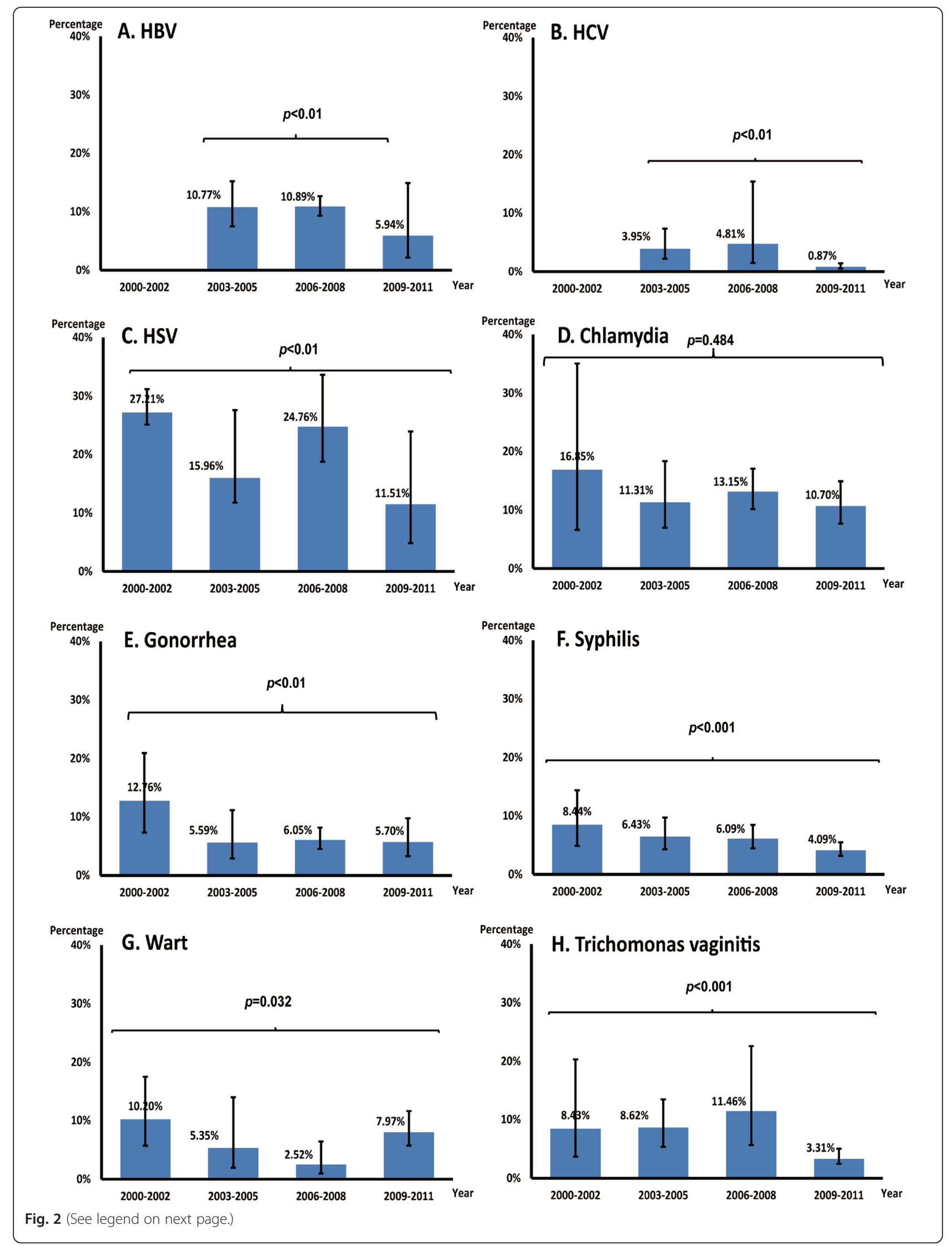


(See figure on previous page.)

Fig. 2 Estimated temporal trend of sexually transmitted infections among female sex workers in China during 2000-2011. *Only infection with > 10 papers were included, so the analysis excluded HPV because there are only four papers being found

We noted a fall in the prevalence of both chlamydia and syphilis over time. The fact that both of these infections are easily treatable and no significant fall has been observed in other viral infections suggest that testing and treatment of STIs either among sex workers or the general community may be the principle factor affecting their fall. On the other hand, syphilis is one of two STIs under sentinel surveillance in China and acquiring more attention from the local CDC. Thus the variation may be attributable to policy changes related to the scalingup of syphilis testing in pregnancy [38] and as part of the 2010-2020 syphilis control plan [39]. Building on declines in chlamydia and syphilis, continued intervention efforts are needed to achieve a decrease in viral infections as well.

HPV is the most prevalent STI among Chinese FSWs $(27.0 \%)$, as well as in the general Chinese population (16.8\%, [24]). Further, HPV prevalence among Chinese FSWs appears to be higher than that found in neighbouring countries $(22.9 \%$ in Thailand [40] and $14.4 \%$ in Singapore [41]), a finding also true for genital warts: $3.3 \%$ in Chinese FSW versus $0.2 \%$ in neighbouring Vietnam [42]. Genital warts are mostly caused by infection with HPV-6 or 11 [43, 44], but more virulent types of HPV (type 16 and 18) are major risk factors for cervical cancers in Chinese women [24, 45, 46]. Co-infection with HPV is also known to facilitate the transmission of chlamydia, gonorrhoea, HSV-2 and HIV [47]. Despite the evidence that early vaccination before sexual debut dramatically reduces the risk of HPV infection, genital warts and cervical cancers among females [48-50], currently there is no national HPV vaccination programme and no specific prevention method for women of reproductive age in China [51]. The majority of Chinese women (approximately $70 \%$ ) have no knowledge about HPV vaccines [52] and even lower levels of knowledge and awareness of HPV in FSW [53, 54]. Given the very high disease burden of HPV, Chinese FSW should be regarded as a priority population for HPV prevention and treatment, especially FSW who are of reproductive age.

A number of limitations should be noted. First, we reported high heterogeneities in our meta-analyses. Despite the efforts of subgroup analysis and meta-regression, significant heterogeneities were still present in subgroups. We have analysed the influences of geographical region, recruitment venue, sample size, publication language, study quality score, and year of publication. Second, statistical power in subgroup analysis was often restricted by the limited number of available studies, especially among the small number of studies from the Northeast. Third, although we only included studies diagnosed with the same methodology, the diagnostic accuracy of tests maybe improved during the study period, so part of the declining trend in prevalence may be owing to the improvement of technology. Fourth, publication bias in the publication language was significant. Fifth, the different clinical diagnostic standards of STIs and hepatitis infections may result in uncertainties in sensitivity and specificity of results.

Our findings illustrated above are consistent with previous reports $[7,55-58]$. We have extended the previous literature by quantifying these risks in a systematic manner to demonstrate the urgency for appropriate prevention and treatment strategies among Chinese FSW, especially those co-infected with HIV. Health resources should be aligned to address these issues. Prevalence of hepatitis infection and STIs in Chinese FSWs is high but stable during the study period 2000-2011. The high prevalence of STIs may relate to the illegal status of sex work. Ongoing research indicates that countries with either regulated or legalized sex work have seen substantial falls in the prevalence of STIs in sex workers, as a result of governments' initiatives for widespread condom programs and promotion of HIV testing and treatment $[59,60]$. China is unlikely to legalize sex work in the near future, therefore comprehensive interventions and HIV programs for FSWs through communitybased organizations should be promoted and prioritised.

\section{Additional files}

Additional file 1: Table S1. Systematic review of 347 studies reporting the prevalence of sexually transmitted infections and/or viral hepatitis infections among female sex workers in China. Table S2. Systematic review of 19 studies reporting the co-infection prevalence of sexually transmitted infections and/or viral hepatitis infections among HIV-positive female sex workers in China. Table S3. Heterogeneity in subgroup meta-analyses. (DOCX $687 \mathrm{~kb}$ )

Additional file 2: PRISMA flow chart for selection of studies. (PNG $159 \mathrm{~kb})$

\section{Competing interests}

The authors declare that they have no competing interests.

Authors' contributions

SS and LZ drafted the manuscript. LZ, JJ, JDT and CKF designed the study. EPFC and XHZ participated in the data collection, data analysis and data extraction. LY participated in the data extraction. JHR participated in the literature search. KEM contributed to manuscript revision. SS and LZ finalized the manuscript. All authors read and approved the final manuscript.

\section{Acknowledgements}

All authors declare that they have no funding received, no current external funding sources for this study and other relationships or activities that could appear to have influenced the submitted work. The Authors would like to 
thank the Research Centre for Public Health, Tsinghua University for the ongoing support for this project.

\begin{abstract}
Author details
${ }^{1}$ School of Public Health and Preventive Medicine, Faculty of Medicine, Nursing and Health Sciences, Monash University, Melbourne, VIC, Australia. ${ }^{2}$ Melbourne Sexual Health Centre, Alfred Health, Melbourne, Australia. ${ }^{3}$ Central Clinical School, Faculty of Medicine, Nursing and Health Sciences, Monash University, Melbourne, VIC, Australia. ${ }^{4}$ The University of North Carolina Project-China, Guangzhou, China. ${ }^{5}$ Department of Health Behaviour, Gillings School of Global Public Health at the University of North Carolina at Chapel Hill, Chapel Hill, USA. ${ }^{6}$ The School of Sociology and Population Studies, Renmin University of China, Beijing, China. 'London School of Hygiene and Tropical Medicine, London, UK. ${ }^{8}$ China Food and Drug Administration Institute of Executive Development, Beijing, China. ${ }^{9}$ Research Center for Public Health, School of Medicine, Tsinghua University, Beijing, China. ${ }^{10}$ The School of Sociology, Tsinghua University, Beijing, China.
\end{abstract}

Received: 19 September 2015 Accepted: 12 December 2015 Published online: 05 January 2016

\section{References}

1. Gottlieb SL, Low N, Newman LM, Bolan G, Kamb M, Broutet N. Toward global prevention of sexually transmitted infections (STIS): the need for STI vaccines. Vaccine. 2014;32(14):1527-35. doi:10.1016/j.vaccine.2013.07.087 [published Online First: Epub Date].

2. Boukli NM, Shetty V, Cubano L, Ricaurte M, Coelho-Dos-Reis J, Nickens Z, et al. Unique and differential protein signatures within the mononuclear cells of HIV-1 and HCV mono-infected and co-infected patients. Clin Proteomics. 2012;9(1):11. doi:10.1186/1559-0275-9-11. [published Online First: Epub Date].

3. Ward H, Ronn M. Contribution of sexually transmitted infections to the sexual transmission of HIV. Curr Opin HIV AIDS. 2010;5(4):305-10. doi:10. 1097/COH.0b013e32833a8844 [published Online First: Epub Date].

4. Strategy to accelerate progress towards the attainment of international development goals and targets related to reproductive health. Reproductive health matters. 2005/07/23 ed. World Health Organisation, 2005:11-8.

5. Reproductive Health Strategy to Accelerate Progress Towards the Attainment of International Development Goals and Targets. World Health Organization, 2014.

6. Prevention and Control of Viral Hepatitis Infection: Framework for Global Action. Secondary Prevention and Control of Viral Hepatitis Infection: Framework for Global Action 2012. http://www.who.int/csr/disease/hepatitis/GHP_framework.pdf.

7. Ding Y, Detels R, Zhao Z, Zhu Y, Zhu G, Zhang B, et al. HIV infection and sexually transmitted diseases in female commercial sex workers in China. J Acquir Immune Defic Syndr. 2005;38(3):314-9.

8. Shuquan Qu WL, Choi K-H, Li R, Jiang D, Zhou Y, Tian F, et al. The Potential for Rapid Sexual Transmission of HIV in China: Sexually Transmitted Diseases and Condom Failure Highly Prevalent Among Female Sex Workers. AIDS Behav. 2002;6(3):267-75.

9. Zhang H, Liao M, Nie X, Pan R, Wang C, Ruan S, et al. Predictors of consistent condom use based on the Information-Motivation-Behavioral Skills (IMB) model among female sex workers in Jinan. China BMC Public Health. 2011;11: 113. doi:10.1186/1471-2458-11-113. [published Online First: Epub Date].

10. Phariss ATD. Women and HIV/AIDS in China. Washington, DC: Freeman Chair China Studies; 2004.

11. Li J, Chen XS, Merli MG, Weir SS, Henderson GE. Systematic differences in risk behaviors and syphilis prevalence across types of female sex workers: a preliminary study in Liuzhou. China Sex Transm Dis. 2012;39(3):195-200. doi: 10.1097/OLQ.0b013e31823d2e2a [published Online First: Epub Date].

12. Chow EP, Muessig KE, Yuan L, Wang Y, Zhang X, Zhao R, et al. Risk behaviours among female sex workers in China: a systematic review and data synthesis. PLoS One. 2015;10(3):e0120595. doi:10.1371/journal.pone. 0120595 [published Online First: Epub Date].

13. Xia X, Luo J, Bai J, Yu R. Epidemiology of hepatitis C virus infection among injection drug users in China: systematic review and meta-analysis. Public Health. 2008;122(10):990-1003. doi:10.1016/j.puhe.2008.01.014 [published Online First: Epub Date].

14. Wu J, Huang J, Xu D, Lu C, Deng X, Zhou X. Infection status and risk factors of HIV, HBV, HCV, and syphilis among drug users in Guangdong. China-a cross-sectional study BMC Public Health. 2010;10:657. doi:10.1186/14712458-10-657 [published Online First: Epub Date].
15. Pan S, Parish WL, Huang Y. Clients of female sex workers: a population-based survey of China. J Infect Dis. 2011;204 Suppl 5:S1211-7. doi:10.1093/infdis/jir537 [published Online First: Epub Date].

16. McLaughlin MM, Chow EP, Wang C, Yang LG, Yang B, Huang JZ, et al. Sexually transmitted infections among heterosexual male clients of female sex workers in China: a systematic review and meta-analysis. PLoS One. 2013;8(8):e71394. doi:10.1371/journal.pone.0071394 [published Online First: Epub Date].

17. Poon AN, Li Z, Wang N, Hong Y. Review of HIV and other sexually transmitted infections among female sex workers in China. AIDS Care. 2011;23 Suppl 1:5-25. doi:10.1080/09540121.2011.554519 [published Online First: Epub Date].

18. Mh B. Guidelines for evaluating prevalence studies. Evid Based Ment Health. 1998;1(2):37-9.

19. Chen P, Yu C, Ruan B, Yang S, Ren J, Xu W, et al. Prevalence of hepatitis B in insular regions of southeast China: a community-based study. PLoS One. 2013;8(2), e56444. doi:10.1371/journal.pone.0056444. [published Online First: Epub Date].

20. Dai S, Shen Z, Zha Z, Leng R, Qin W, Wang C, et al. Seroprevalence of HIV, syphilis, and hepatitis $C$ virus in the general population of the Liangshan Prefecture, Sichuan Province. China J Med Virol. 2012;84(1):1-5. doi:10.1002/ jmv.22214 [published Online First: Epub Date].

21. Hesketh T, Ye X, Zhu W. Syphilis in China: the great comeback. Emerg Health Threats J. 2008;1:e6. doi:10.3134/ehtj.08.006 [published Online First: Epub Date]

22. Lu F, Jia Y, Bin S, Li C, Limei S, Kristensen S, et al. Predictors for casual sex and/or infection among sexually transmitted disease clinic attendees in China. Int J STD AIDS. 2009;20(4):241-8. doi:10.1258/ijsa.2008.008290 [published Online First: Epub Date].

23. Parish WL, Laumann EO, Cohen MS, Pan S, Zheng H, Hoffman I, et al. Population-based study of chlamydial infection in China: a hidden epidemic. JAMA. 2003;289(10):1265-73.

24. Zhao FH, Lewkowitz AK, Hu SY, Chen F, Li LY, Zhang QM, et al. Prevalence of human papillomavirus and cervical intraepithelial neoplasia in China: a pooled analysis of 17 population-based studies. Int J Cancer. 2012;131(12): 2929-38. doi:10.1002/ijc.27571 [published Online First: Epub Date].

25. Begg CB, Mazumdar M. Operating characteristics of a rank correlation test for publication bias. Biometrics. 1994;50(4):1088-101.

26. Luo Z, Xie Y, Deng M, Zhou X, Ruan B. Prevalence of hepatitis B in the southeast of China: a populationbased study with a large sample size. Eur J Gastroenterol Hepatol. 2011;23(8):695-700. doi:10.1097/MEG. ob013e328347322b [published Online First: Epub Date].

27. Cdc C. National epidemiological investigation of HBV in China Secondary National epidemiological investigation of HBV in China. 2008. http://www. chinacdc.cn/n272442/n272530/n3246177/23316.html.

28. Catherine F. Houlihana NLL, Deborah Watson-Jonesa, Karen K. Smith-McCuned, Stephen Shiboskie, Patti E. Gravittf, Jennifer S. Smithh, Louise Kuhni, Chunhui Wangi and Richard Hayes. Human papillomavirus infection and increased risk of HIV acquisition. A systematic review and meta-analysis. AIDS 2012;26:2211-22.

29. Yao JL. Perinatal transmission of hepatitis B virus infection and vaccination in China. Gut. 1996:38 Suppl 2:S37-8.

30. Chen CJ, Wang LY, Yu MW. Epidemiology of hepatitis B virus infection in the Asia-Pacific region. J Gastroenterol Hepatol. 2000;15(Suppl):E3-6.

31. Ping H. [Status Analysis of epidemic trend of Hepaitis B in Guang Dong]. Chin Prev Med. 2002;3(2):105-7.

32. Dai Zhi Cheng QGM. The investigation of Chinese hepatitis epidemiological. Bei Jing: Science and Technology Press; 1997.

33. Liang X, Bi S, Yang W, Wang L, Cui G, Cui F, et al. Evaluation of the impact of hepatitis B vaccination among children born during 1992-2005 in China. J Infect Dis. 2009;200(1):39-47. doi:10.1086/599332 [published Online First: Epub Date].

34. UNODC. World Drug Report. Vienna: United Nations Office on Drugs and Crime; 2015.

35. Tyndall MW, Patrick D, Spittal P, Li K, O'Shaughnessy MV, Schechter MT. Risky sexual behaviours among injection drugs users with high HIV prevalence: implications for STD control. Sex Transm Infect. 2002;78 Suppl 1:1170-5.

36. Lin Peng YF, Fan Zi Fan, Mai Xin Rong, Wang Ye, Liu Yong Ying, Fu Xiao Bing, Zhao Qian Qian, He Qun, Xu Rui Heng. TThe qualitive analysis of injected drug users behaviours among Guang Dong]. South China J Prev Med. 2006;1:23-26.

37. Pei Dong nu YB, Lai Sheng Zheng, Wang Fang Qian. [High risk behaviour investigation and syphilis infection survelliance among female sex workers and drug users in Hainan]. Chin J STD. 2002;8(3):160-61. 
38. Tucker JD, Hawkes SJ, Yin YP, Peeling RW, Cohen MS, Chen XS. Scaling up syphilis testing in China: implementation beyond the clinic. Bull World Health Organ. 2010;88(6):452-7. doi:10.2471/blt.09.070326 [published Online First: Epub Date].

39. Tucker JD, Cohen MS. China's syphilis epidemic: epidemiology, proximate determinants of spread, and control responses. Curr Opin Infect Dis. 2011 24(1):50-5. doi:10.1097/QCO.0b013e32834204bf [published Online First: Epub Date].

40. Chandeying V, Garland SM, Tabrizi SN. Prevalence and typing of human papilloma virus (HPV) among female sex workers and outpatient women in southern Thailand. Sex Health. 2006;3(1):11-4.

41. Chan R, Khoo L, Ho TH, Koh CF, Lee IW, Yam KL, et al. A comparative study of cervical cytology, colposcopy and PCR for HPV in female sex workers in Singapore. Int J STD AIDS. 2001;12(3):159-63.

42. Lan PT, Lundborg CS, Phuc HD, Sihavong A, Unemo M, Chuc NT, et al Reproductive tract infections including sexually transmitted infections: a population-based study of women of reproductive age in a rural district of Vietnam. Sex Transm Infect. 2008;84(2):126-32. doi:10.1136/sti.2007.027821 [published Online First: Epub Date].

43. Chang L, Ci P, Shi J, Zhai K, Feng X, Colombara D, et al. Distribution of genital wart human papillomavirus genotypes in China: a multi-center study. J Med Virol. 2013;85(10):1765-74. doi:10.1002/jmv.23646 [published Online First: Epub Date].

44. Wu XL, Zhang CT, Zhu XK, Wang YC. Detection of HPV types and neutralizing antibodies in women with genital warts in Tianjin City. China Virol Sin. 2010:25(1):8-17. doi:10.1007/s12250-010-3078-4 [published Online First: Epub Date].

45. Bian ML, Cheng JY, Ma L, Cong X, Liu J, Chen Y, et al. Evaluation of the detection of 14 high-risk human papillomaviruses with HPV 16 and HPV 18 genotyping for cervical cancer screening. Exp Ther Med. 2013;6(5):1332-6. doi:10.3892/etm.2013.1309 [published Online First: Epub Date].

46. Li J, Zhang D, Zhang Y, Wang $X$, Lin Y, Hu L. Prevalence and genotype distribution of human papillomavirus in women with cervical cancer or high-grade precancerous lesions in Chengdu, western China. Int I Gynaecol Obstet. 2011;112(2):131-4. doi:10.1016/j.ijgo.2010.08.010 [published Online First: Epub Date].

47. Houlihan CF, Larke NL, Watson-Jones D, Smith-McCune KK, Shiboski S, Gravitt PE, et al. Human papillomavirus infection and increased risk of HIV acquisition. A systematic review and meta-analysis. AIDS (London, England). 2012;26(17):2211-22. doi:10.1097/QAD.0b013e328358d908. [published Online First: Epub Date].

48. Drolet M, Benard E, Boily MC, Ali H, Baandrup L, Bauer H et al. Populationlevel impact and herd effects following human papillomavirus vaccination programmes: a systematic review and meta-analysis. The Lancet Infectious diseases. 2015. doi:10.1016/s1473-3099(14)71073-4 [published Online First: Epub Date].

49. Harrison C, Britt H, Garland S, Conway L, Stein A, Pirotta M, et al. Decreased management of genital warts in young women in australian general practice post introduction of national HPV vaccination program: results from a nationally representative cross-sectional general practice study. PLoS One. 2014;9(9):e105967. doi:10.1371/journal.pone.0105967 [published Online First: Epub Date]

50. Chow EP, Read TR, Wigan R, Donovan B, Chen MY, Bradshaw CS, et al. Ongoing decline in genital warts among young heterosexuals 7 years after the Australian human papillomavirus (HPV) vaccination programme. Sex Transm Infect. 2015:91(3):214-9. doi:10.1136/sextrans-2014-051813 [published Online First: Epub Date]

51. Zhu FC, Chen W, Hu YM, Hong Y, Li J, Zhang X, et al. Efficacy, immunogenicity and safety of the HPV-16/18 AS04-adjuvanted vaccine in healthy Chinese women aged 18-25 years: results from a randomized controlled trial. Int J Cancer. 2014;135(11):2612-22.

52. Zeng Jing GQ. Cognitive situation and health education demand of Chinese women to human papillomavirus and its vaccine. Chin Nurs Res. 2013; 27(6B):1673-4.

53. Hong Y, Zhang C, Li X, Lin D, Liu Y. HPV and cervical cancer related knowledge, awareness and testing behaviors in a community sample of female sex workers in China. BMC Public Health. 2013;13:696. doi:10.1186/ 1471-2458-13-696 [published Online First: Epub Date].

54. Li HM, Liang GJ, Yin YP, Wang QQ, Zheng ZJ, Zhou JJ, et al. Prevalence and genotype distribution of human papillomavirus infection among female sex workers in Guangxi, China: implications for interventions. J Med Virol. 2012; 84(5):798-803. doi:10.1002/jmv.23264 [published Online First: Epub Date].

55. Li Y, Detels R, Lin P, Fu X, Deng Z, Liu Y, et al. Difference in risk behaviors and STD prevalence between street-based and establishment-based FSWs in Guangdong Province, China. AIDS Behav. 2012;16(4):943-51. doi:10.1007/ s10461-011-0102-0 [published Online First: Epub Date].

56. Van den Hoek A, Yuliang F, Dukers NH, Zhiheng C, Jiangting F, Lina Z, et al. High prevalence of syphilis and other sexually transmitted diseases among sex workers in China: potential for fast spread of HIV. AIDS (London, England). 2001;15(6):753-9.

57. Fan YG, Liu JJ, Zhang YJ, Dai SY, Li MQ, Ye DQ. HIV, other sexually transmitted infections, and risk behaviors among female sex workers in Liuzhou, China. Int J Gynaecol Obstet. 2015;128(1):18-22. doi:10.1016/j.ijgo. 2014.07.024 [published Online First: Epub Date].

58. Jin X, Chan S, Ding G, Wang H, Xu J, Wang G, et al. Prevalence and risk behaviours for Chlamydia trachomatis and Neisseria gonorrhoeae infection among female sex workers in an HIV/AIDS high-risk area. Int J STD AIDS. 2011;22(2):80-4

59. Lee DM, Binger A, Hocking J, Fairley CK. The incidence of sexually transmitted infections among frequently screened sex workers in a decriminalised and regulated system in Melbourne. Sex Transm Infect. 2005; 81(5):434-6. doi:10.1136/sti.2004.014431 [published Online First: Epub Date].

60. Harcourt C, Egger S, Donovan B. Sex work and the law. Sex Health. 2005; 2(3):121-8.

61. Liu SS, Chan KY, Leung RC, Chan KK, Tam KF, Luk MH, et al. Prevalence and risk factors of Human Papillomavirus (HPV) infection in southern Chinese women - a population-based study. PLoS One. 2011;6(5):e19244. doi:10. 1371/journal.pone.0019244 [published Online First: Epub Date].

62. Wang X, Gu D, Lou B, Xu B, Qian F, Chen Y. Hospital-based prevalence of high-risk cervical HPV types infecting the general population and female sex workers in Huzhou. China Int J Gynaecol Obstet. 2013;120(1):37-41. doi: 10.1016/j.ijgo.2012.07.019 [published Online First: Epub Date]

\section{Submit your next manuscript to BioMed Central and we will help you at every step:}

- We accept pre-submission inquiries

- Our selector tool helps you to find the most relevant journal

- We provide round the clock customer support

- Convenient online submission

- Thorough peer review

- Inclusion in PubMed and all major indexing services

- Maximum visibility for your research

Submit your manuscript at www.biomedcentral.com/submit 